\title{
Objetivos de Desarrollo Sostenible: impacto de la falta de protección financiera en salud en países de América Latina y el Caribe
}

\author{
Camilo Cid ${ }^{1}$, Gabriela Flores ${ }^{2}$, Amalia Del Riego ${ }^{1}$ y James Fitzgerald ${ }^{1}$
}

Forma de citar

Cid C, Flores G, Del Riego A, Fiztgerald J. Objetivos de Desarrollo Sostenible: impacto de la falta de protección financiera en salud en países de América Latina y el Caribe. Rev Panam Salud Publica. 2021;45:e95. https://doi.org/10.26633/ RPSP.2021.95

RESUMEN

Objetivo. Determinar el impacto del gasto de bolsillo en salud en los hogares, los niveles de protección financiera y su desigualdad según variables relevantes en países de la Región. Se indaga su evolución y relación con el uso de servicios del sistema de salud.

Métodos. Se recopila información descriptiva comparada acerca del gasto de bolsillo, su incidencia en la población, y su peso y composición en el consumo de los hogares. Se presentan indicadores de protección financiera en el nivel nacional y su distribución por quintiles de consumo total de hogares y por género. Se contrastan con un indicador de cobertura de servicios.

Resultados. Los indicadores de gasto de bolsillo y protección financiera son deficientes pero diferenciados entre los países. Se identifica la composición del gasto en salud para un subgrupo de ellos y existen gradientes significativos cuando se estudian las desigualdades. Para algunos casos, se muestran cambios en el tiempo y posibles asociaciones con los niveles de cobertura de servicios.

Discusión. La desprotección financiera afecta a una gran parte de la población, se configuran grupos de países con dificultades mayores que otros, con preponderancia de gasto en medicamentos y exposición mayor de grupos en situaciones de vulnerabilidad, como los más pobres y las mujeres, lo que denota una gran inequidad. Se identifican políticas de algunos países que pueden asociarse con la evolución de la protección financiera. Para reemplazar el gasto de bolsillo, barrera para el acceso, los países necesitan aumentar el gasto público mediante el financiamiento de los sistemas de salud en transformación hacia la salud universal.

Palabras clave Gastos en salud; protección contra riesgos financieros; gasto catastrófico en salud; gasto empobrecedor en salud; financiación de la atención de salud.

Los informes de protección financiera en salud presentados durante la Asamblea General de Naciones Unidas en el 2019, como parte del monitoreo de los Objetivos de Desarrollo Sostenible (ODS) $(1,2)$, indicaron que el gasto de bolsillo en salud (GBS) representaba más de $10 \%$ del presupuesto del hogar para 927 millones de personas, y 10\% de ellas vivían en América Latina y el Caribe (ALC). Asimismo, 183 millones de personas se empobrecieron a causa de los pagos directos en salud; de las cuales 11,5 millones vivían en ALC.

Este trabajo profundiza en la situación de protección financiera en salud en países de la Región, sobre la base de que la insuficiencia de esta protección representa un impedimento significativo para el acceso a la salud y la equidad, y contraviene los compromisos asumidos en los niveles global y regional,

\footnotetext{
${ }^{1}$ Organización Panamericana de la Salud. Washington D.C., Estados Unidos de

América. $\bowtie$ Camilo Cid, cidcam@paho.org
}

${ }^{2}$ Organización Mundial de la Salud, Ginebra, Suiza.

Este es un artículo de acceso abierto distribuido bajo los términos de la licencia Creative Commons Attribution-NonCommercial-NoDerivs 3.0 IGO, que permite su uso, distribución y reproducción en cualquier medio, siempre que el trabajo original se cite de la manera adecuada. No se permiten modificaciones a los artículos ni su uso comercial. Al reproducir un artículo no debe haber ningún indicio de que la OPS o el artículo avalan a una organización o un producto específico. El uso del logo de la OPS no está permitido. Esta leyenda debe conservarse, junto con la URL original del artículo. Crédito del logo y texto open access: PLoS, bajo licencia Creative Commons Attribution-Share Alike 3.0 Unported. 
tales como la estrategia de salud universal de la Organización Panamericana de la Salud (OPS) (3), los ODS (4) e hitos relacionados (5-7). En estos compromisos, los países asumen el reto de lograr que las personas y las comunidades tengan acceso a los servicios de salud integrales y de calidad que necesitan sin dificultades financieras.

El GBS se define como el pago directo requerido en el momento de la utilización de servicios y en el punto de acceso, neto de cualquier reembolso posterior (8). El hecho que estos pagos constituyan un requisito para recibir la atención los convierte en una barrera de acceso. Aunque sean mínimos, solo necesitan ser suficientemente elevados, en relación con la capacidad de pago de los hogares, para ser relevantes.

Para aquellos hogares que movilizan esos recursos económicos, incurrir en estos gastos puede afectar de manera negativa su nivel de bienestar y el consumo de otros bienes y servicios básicos como alimentos, vivienda o educación. De este modo, el GBS puede representar una proporción del consumo total de los hogares ${ }^{1}$ demasiado alta; es decir, ser un gasto catastrófico, e incluso puede arrastrar a los hogares a la pobreza, mantenerlos en ella o profundizarla, en cuyo caso se trata de un gasto empobrecedor. Por otro lado, las personas y familias que no puedan pagar por los servicios, lo que se suma a otras barreras existentes (9-11), tendrán una de sus necesidades básicas, la de la salud, insatisfecha, lo que además puede llevar a la profundización de los problemas económicos. Las dificultades generadas por la falta de protección financiera relacionadas con el uso de los servicios de salud pueden ser un problema en cualquier país y generar dificultades económicas para todo tipo de hogar, desde los de mayor ingreso, a los más pobres. Además, puede exacerbar las desigualdades socioeconómicas entre la población, lo que puede ser especialmente cierto para grupos en situación de vulnerabilidad.

En base a resultados para un grupo de países, este trabajo busca: a) determinar y comparar el impacto del GBS en los hogares, así como su composición; b) mostrar los niveles de catástrofe financieras y empobrecimiento que produce entre los hogares, y cómo se distribuye por género y por niveles de bienestar; y c) asociar la evolución de los indicadores de protección financiera con los sistemas de salud existentes y sus niveles de cobertura de servicios.

\section{MATERIALES Y MÉTODOS}

Se comienza por calcular la incidencia del GBS; es decir, el porcentaje de población que realiza algún pago de esta índole en cada país. A su vez, se presenta la porción del consumo de los hogares que se dedica a gastos en salud y, para algunos casos, se informa la composición del GBS, esto es, si el objeto de gasto es servicios (ambulatorios u hospitalarios) o medicamentos.

Luego se muestran los indicadores de gasto catastrófico y gasto empobrecedor en salud de los países. Estos requieren información sobre el GBS en el numerador, y el gasto en consumo total del hogar, como medida de su bienestar, en el denominador. La incidencia o tasa de gasto catastrófico en salud se define como la proporción de la población con un GBS superior a $10 \%$ o al $25 \%$ del consumo total del hogar (1213). Para los hogares más ricos, gastar más de una cuarta parte

\footnotetext{
${ }^{1}$ En este trabajo se utilizó el consumo total del hogar como la variable de bienestar sobre la cual se mide el impacto del GBS, equivalente a los conceptos de presupuesto familiar o capacidad de pago.
}

de su capacidad de consumo en salud podría llevar a recortar el consumo de otras necesidades, pero no necesariamente a niveles inferiores a los necesarios para subsistir. Para los hogares más pobres, gastar menos de $10 \%$ de su presupuesto en salud puede comprometer su esfuerzo por alcanzar un nivel de vida digno.

La incidencia o tasa de gasto empobrecedor en salud se entiende como la proporción de la población que se empobrece por efecto del GBS; es decir, es el porcentaje que vive en hogares en los que este gasto, al ser sustraído, deja al gasto en consumo por debajo de la línea de pobreza. Por otra parte, se utiliza el indicador de aumento en la profundidad de la pobreza debido al GBS, que se mide como los cambios en la brecha de pobreza cuando el GBS del hogar se incluye o se excluye del consumo total, y da cuenta de los hogares que cruzan los umbrales debido a GBS y de aquellos que se empobrecen aún más con este gasto. Este efecto no es visible en el indicador de incidencia de empobrecimiento $(1,14,15)$.

Para los cálculos de los indicadores de gasto empobrecedor en salud se utilizan tres líneas de pobreza: a) la pobreza extrema, definida como vivir con 1,90 dólares estadounidenses por día, que corresponde a la línea de los países de bajos ingresos (16); b) una línea de pobreza de 3,20 dólares por día, que corresponde al estándar utilizado para evaluar países de ingresos medios bajos (17); y c) una línea de pobreza relativa equivalente a $60 \%$ de la mediana del consumo per cápita diario del hogar, que captura el impacto del GBS sobre la pobreza en todos los grupos de países. Las líneas monetarias se establecen en la paridad de poder adquisitivo (PPA) de 2011.

Para los cálculos de incidencia y composición del GBS, así como para los análisis de desigualdad de género y según quintiles de consumo total de los hogares, se utilizaron las bases de datos de las encuestas de los países (ver el cuadro A1, en el material suplementario), procesadas mediante STATA ${ }^{\circledR}$. Para mostrar el impacto financiero del GBS en los hogares y los indicadores de protección financiera de los países, se utilizó información de la actualización mundial de protección contra los riesgos financieros para los ODS, publicada por la Organización Mundial de la Salud (OMS) y el Banco Mundial $(1,2,18,19)$. Para el análisis cruzado entre la cobertura de servicios y la protección financiera, se incorporó el índice de cobertura de servicios de la OMS (2), que se presenta para el último año disponible y, en algunos casos, para el período coincidente con los de protección financiera. También se usaron los datos oficiales de cuentas de salud de la OMS (20).

\section{RESULTADOS}

\section{Impacto del gasto de bolsillo en salud en los hogares}

En la figura 1 se muestra la incidencia del GBS para diez países (figura 1a). Existen casos en que solo alrededor de la mitad de la población realiza GBS, como México y Suriname, y otros donde el GBS muestra una alta incidencia y está presente en más de $80 \%$ de la población, como Chile, Ecuador y Perú. Por otra parte, la porción del consumo de los hogares que se destina a GBS puede variar desde aproximadamente $0,7 \%$ en El Salvador hasta $5,2 \%$ en el caso de Barbados, para los 16 países de la figura $1 b$. 
FIGURA 1. Incidencia del gasto de bolsillo en salud y su proporción respecto del gasto total de los hogares. A: porcentaje de la población que realiza gasto de bolsillo en salud (10 países). B: porcentaje del presupuesto de los hogares dedicado a gasto de bolsillo en salud (16 países).

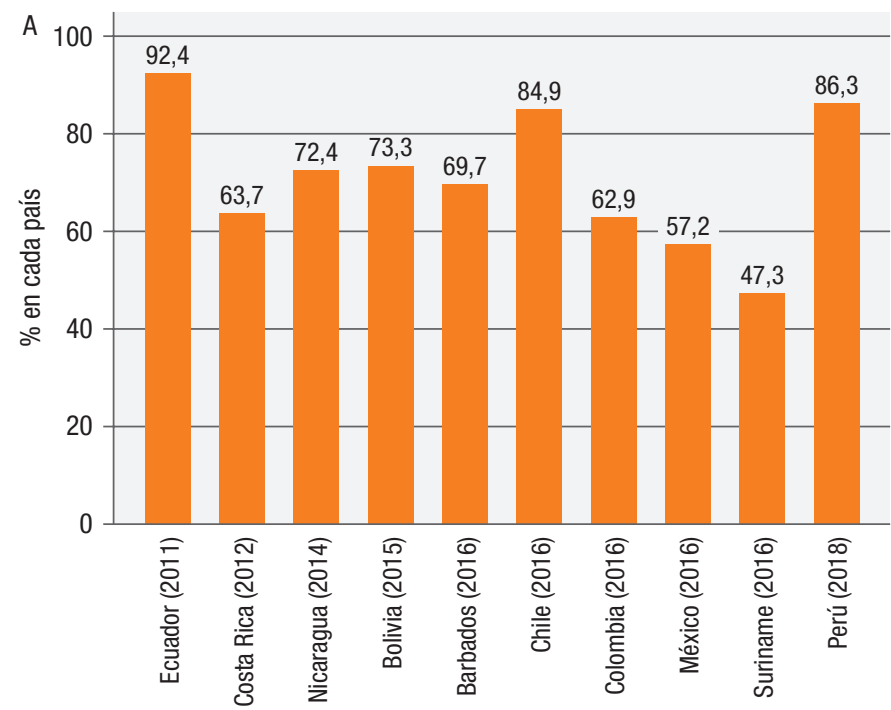

Fuente: elaborada a partir de datos producidos por la Organización Panamericana de la Salud para el año más reciente en cada país.

Existe una asociación entre la incidencia del GBS y la porción del consumo del hogar que este absorbe ${ }^{2} y$, al analizar ambas figuras, se establecen al menos dos tipos de situaciones. Por una parte, en dos países con similar incidencia de GBS, como Nicaragua y Bolivia (72,4\% y 73,3\%, respectivamente), el impacto del GBS en el consumo de los hogares es diferente, mucho mayor en el país de ingreso más bajo (casi dos veces más alto en Nicaragua que en Bolivia ${ }^{3}$ ).

Por otra parte, las diferencias observadas en la extensión y la proporción en el consumo del hogar denotan distintas composiciones del GBS en los países. Este es el caso de Barbados y Chile, ambos con un promedio del consumo del hogar absorbido por el GBS casi similar (4,8\% y 5,2\%, respectivamente). Sin embargo, los pagos en medicamentos absorben 53\% del GBS para el $69,7 \%$ de la población en Barbados; mientras que, en Chile, solo $36,3 \%$ del GBS se destina a medicamentos para $84,9 \%$ de población que presenta GBS.

La importancia del gasto en medicamentos en Barbados no es una excepción. Por el contrario, es el ítem más importante dentro del GBS de los hogares en muchos países (cuadro 1). Incluso en algunos países como Bolivia, Colombia, Ecuador y México, es mayor que 60,0\%, seguidos por el GBS por servicios ambulatorios. En el caso de Perú, los medicamentos también son la principal categoría de GBS, sin llegar a superar la mitad de ellos. Chile es una excepción, dado que son los GBS por servicios ambulatorios los que representan la categoría principal, con $49,3 \%$. Estas cifras suponen un porcentaje menor de pagos por servicios hospitalarios en promedio, para todos estos casos.

${ }^{2}$ Correlación monotónica positiva con coeficiente de correlación de Spearman $\rho(23)=0,35 ; \mathrm{P}=0.09$

${ }^{3}$ Según datos del Banco Mundial, en 2015, el producto interno bruto per cápita del Estado Plurinacional de Bolivia era 35\% más alto que el de Nicaragua. Para más información, véase: https://data.worldbank.org/indicator/NY.GDP.PCAP. PP.CD?locations $=\mathrm{BO}-\mathrm{NI}$

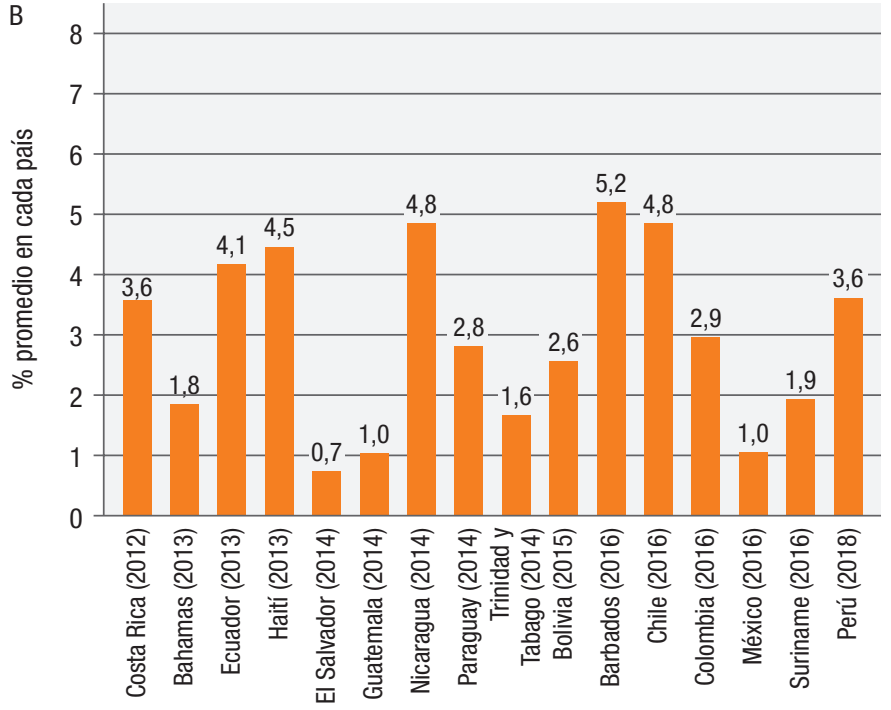

Fuente: elaborada a partir de la actualización del 2019 de los datos publicados en las bases de datos de la Organización Mundial de la Salud y el Banco Mundial (estimaciones más recientes para cada país).

CUADRO 1. Gasto de bolsillo en medicamentos y servicios ambulatorios en porcentaje del gasto total de bolsillo de los hogares (promedio por país) en siete países de la Región

\begin{tabular}{lccc}
\multicolumn{1}{c}{ País (año) } & $\begin{array}{c}\text { Pagos por } \\
\text { medicamentos }\end{array}$ & $\begin{array}{c}\text { Pagos por } \\
\text { servicios } \\
\text { ambulatorios }\end{array}$ & $\begin{array}{c}\text { Total de gasto en } \\
\text { medicamentos y } \\
\text { servicios ambulatorios }\end{array}$ \\
Bolivia (2015) & $74,2 \%$ & $19,9 \%$ & 94,10 \\
Barbados (2016) & $53,0 \%$ & $46,7 \%$ & $100,0 \%$ \\
Chile (2016) & $36,3 \%$ & $49,3 \%$ & $85,6 \%$ \\
Colombia (2016) & $68,5 \%$ & $18,6 \%$ & $87,1 \%$ \\
Ecuador (2011) & $61,2 \%$ & $24,1 \%$ & $85,3 \%$ \\
México (2016) & $73,0 \%$ & $24,9 \%$ & $97,9 \%$ \\
Perú (2017) & $48,0 \%$ & $25,0 \%$ & $73,0 \%$ \\
\hline
\end{tabular}

aEn Barbados, los pagos por servicios ambulatorios incluyen también gasto por servicios hospitalarios de internación Fuente: datos provisionales de la Organización Panamericana de la Salud, obtenidos en el Taller de estimación
de indicadores de protección financiera. Washington D.C.: OPS; 2019. Los cálculos se obtuvieron de la última encuesta de gasto de hogares disponible de cada país.

\section{Nivel de la protección financiera y comparación entre países}

La población sometida a gasto catastrófico en salud puede alcanzar desde $0,2 \%$ en México hasta $16,4 \%$ en Barbados (figura $2 a$ ). La mayoría de los países (diez de ellos) presenta una incidencia del gasto catastrófico para el 5,0\% de la población o más, al considerar el umbral del 10\% de consumo total del hogar (figura 2a). Además, para cinco de esos países, la incidencia de gasto catastrófico es muy elevada, con más de $10,0 \%$ de la población comprometida. Se trata de aquellos que presentan también mayor impacto en el consumo de los hogares ${ }^{4}$ : Barbados, Chile, Ecuador, Haití y

${ }^{4}$ El GBS como proporción del consumo total de los hogares presenta una relación con el gasto catastrófico en salud con un coeficiente de Spearman $\rho(23)=0,98$ y 0,89 al umbral del $10 \%$ y el $25 \%$, respectivamente, $\mathrm{P}=0$ para los dos umbrales. 
FIGURA 2. Protección financiera en 16 países de América Latina y el Caribe: gasto catastrófico en salud de los hogares y gasto empobrecedor en salud de los hogares. A. incidencia del gasto catastrófico en salud (indicador ODS 3.8.2). B: incidencia del gasto empobrecedor en salud.

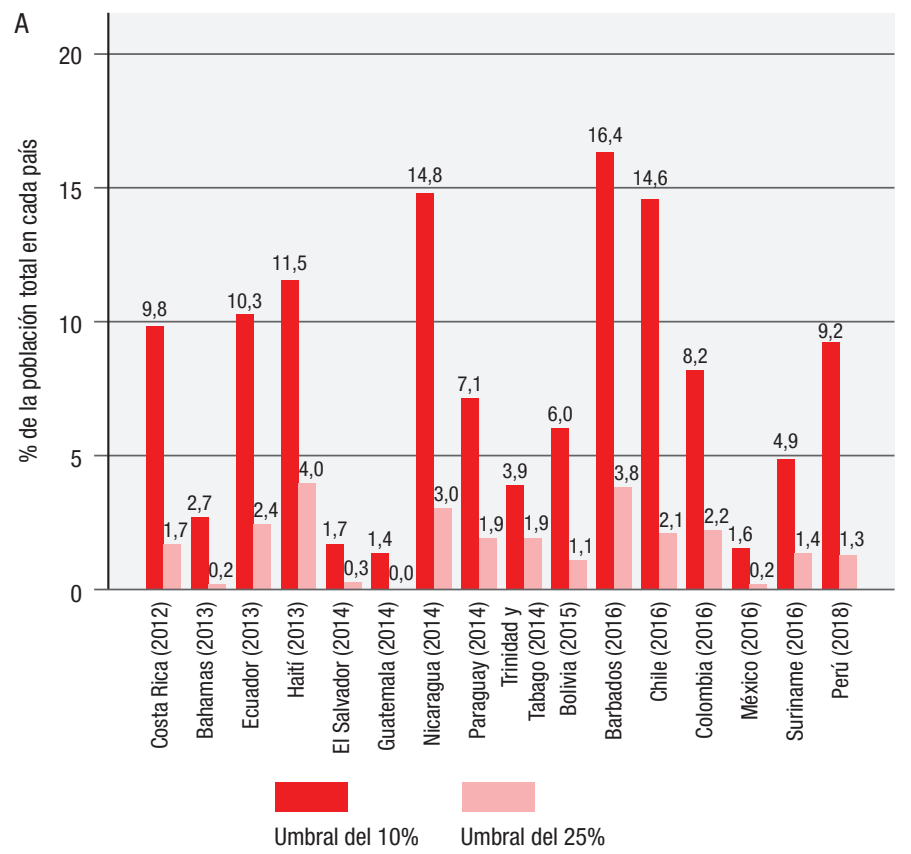

Nota: el indicador ODS 3.8 .2 identifica la proporción de la población con gasto de bolsillo en salud superiores a $10 \%$ o $25 \%$ del presupuesto del hogar. El presupuesto del hogar se mide con el consumo total del hogar.

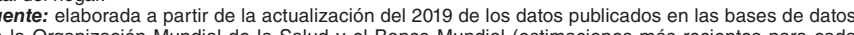
de la Organización Mundial de la Salud y el Banco Mundial (estimaciones más recientes para cada país).

Nicaragua. Luego, otros cinco países muestran un resultado que va desde $5 \%$ a $10 \%$ de la población comprometida, y seis países presentan valores por debajo del $5 \%$ de la población.

El indicador de gasto catastrófico al umbral de 25\% representan un subconjunto de los que se ven afectados al umbral anterior. En este umbral, es posible observar los mismos grupos de países, aunque en distinto orden. Los que presentaban más de $10 \%$ de población comprometida, oscilan ahora entre $2,1 \%$ (Chile) y 4\% (Haití) de la población. En el grupo de impacto de entre $5 \%$ y $10 \%$ de la población con el umbral anterior, se mueven entre 1,1\% (Bolivia) y 2,2\% (Colombia). En el grupo de impacto menor, ahora la incidencia va desde una prácticamente nula (Guatemala), a 1,9\% de la población (Trinidad y Tabago).

En cuanto al gasto empobrecedor en salud (figura 2b), su incidencia al umbral de 3,2 dólares puede involucrar desde niveles promedio casi nulos (Bahamas y Suriname) hasta más de $4 \%$ de la población que es empujada a la pobreza por GBS, como sucede en Haití. Si se considera el umbral de pobreza relativa, la mayoría de los países muestra tasas de empobrecimiento por sobre el 1,0\% de la población. Los cinco países que presentan mayor incidencia de empobrecimiento por gastos en salud son los mismos que presentan mayores gastos catastróficos, con variaciones en el orden. En este caso, presentan resultados que van desde 1,8\% de la población en empobrecimiento como en Barbados, hasta el caso de Haití, que dobla ese porcentaje.

El cuadro 2 muestra que el aumento de la brecha de pobreza debido al GBS varía entre menos del 0,2\% en el Salvador, Guatemala y México, hasta más del 1,0\% en Paraguay y Haití. No

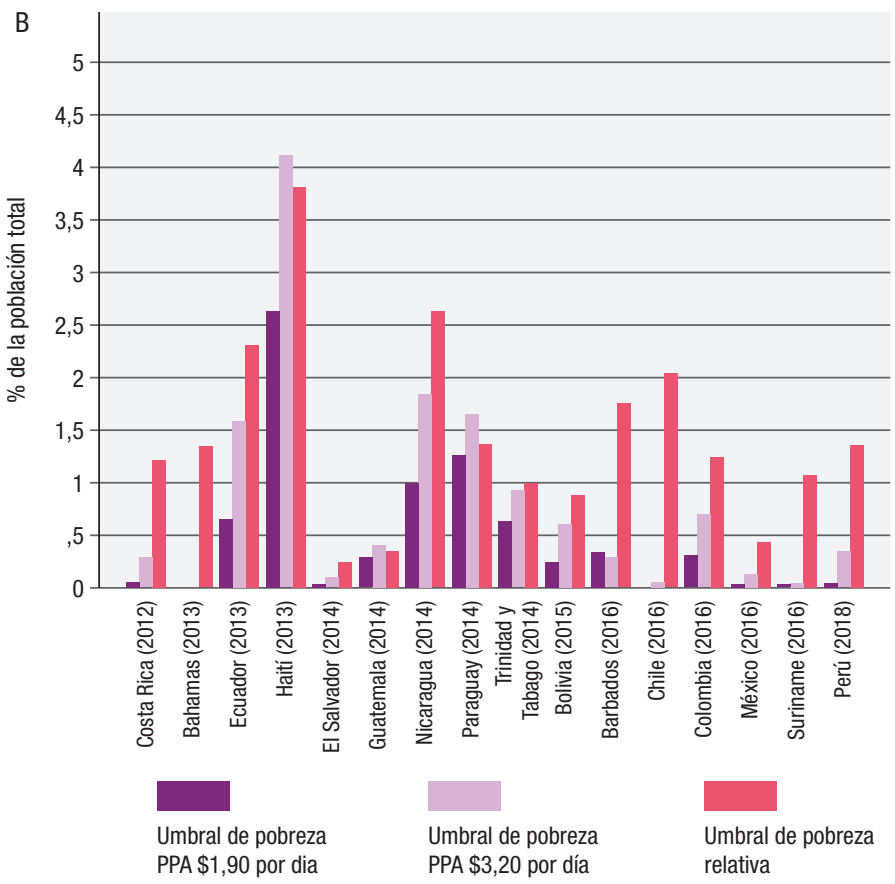

Nota: el umbral de pobreza relativa corresponde al $60 \%$ de la mediana del consumo total de los hogares por día en cada país. Los umbrales de 1,90 dólares estadounidenses (USD) y USD 3,20 por día se miden en paridad de poder adquisitivo (PPA), el equivalente en moneda local se encuentra en la base de datos PovcalNet del banco mundial (actualización de marzo de 2019). La incidencia indica el porcentaje de la población en cada país que termina por debajo del umbral de pobreza absoluto o relativo por causa del
gasto directo en salud. gasto directo en salud.

Fuente: elaborada a partir de la actualización del 2019 de los datos publicados en las bases de datos de la Organización Mundial de la Salud y el Banco Mundial (estimaciones más recientes para cada país).

CUADRO 2. Aumento en la profundidad de la pobreza debido al gasto de bolsillo en salud, umbral relativo equivalente a $60 \%$ de la mediana del consumo

\begin{tabular}{lc}
\multicolumn{1}{c}{ País (año más reciente) } & $\begin{array}{c}\text { Como proporción de una línea de } \\
\text { pobreza relativa }(60 \% \text { de la mediana del } \\
\text { consumo o ingreso per cápita diario) } \\
\text { (aumento en puntos porcentuales) }\end{array}$ \\
El Salvador (2014) & 0,1 \\
Guatemala (2014) & 0,1 \\
México (2016) & 0,2 \\
Bahamas (2013) & 0,3 \\
Suriname (2016) & 0,3 \\
Bolivia (2015) & 0,3 \\
Barbados (2016) & 0,5 \\
Chile (2016) & 0,5 \\
Costa Rica (2012) & 0,5 \\
Colombia (2016) & 0,5 \\
Nicaragua (2014) & 0,9 \\
Ecuador (2013) & 1,0 \\
Paraguay (2014) & 1,3 \\
Haití (2013) & 1,9 \\
\hline
\end{tabular}

Fuente: elaborado a partir de la actualización del 2019 de los datos publicados en las bases de datos de la Organización Mundial de la Salud y el Banco Mundial (estimaciones más recientes para cada país).

necesariamente una mayor brecha de pobreza coincide con tasas más altas de empobrecimiento por GBS. Por ejemplo, Paraguay tiene casi la mitad de la tasa de empobrecimiento por GBS que 
FIGURA 3. Desigualdades por sexo del jefe del hogar en la protección financiera en salud en 7 países de América Latina y el Caribe. A: incidencia del gasto catastrófico en salud según sexo del jefe del hogar (indicador ODS 3.8.2, umbral del 10\%). B: incidencia del gasto empobrecedor en salud (umbral de pobreza de \$1,90 por día). C: incidencia del gasto empobrecedor en salud (umbral de pobreza relativa).

A

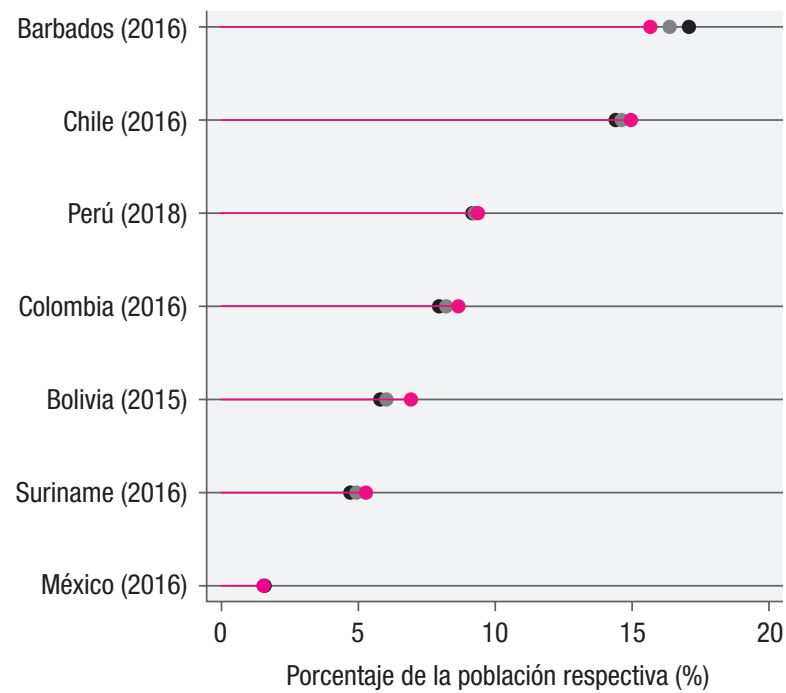

Nota: el indicador ODS 3.8.2 identifica la proporción de la población con gasto de bolsillo en salud superiores a $10 \%$ ० $25 \%$ del presupuesto del hogar. El presupuesto del hogar se mide con el consumo

Fuente: elaborada a partir de datos producidos por la Organización Panamericana de la Salud, sobre la base de las estimaciones más recientes para cada país.

C

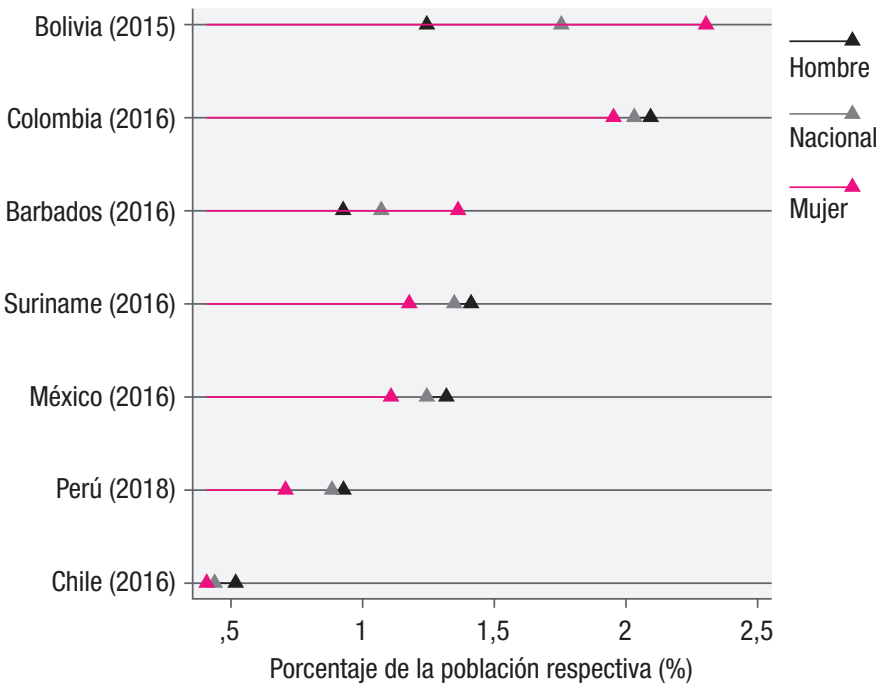

Nota: el umbral de pobreza relativa corresponde a $60 \%$ de la mediana del consumo total de los hogares por día en cada país. La incidencia indica el porcentaje de la población en cada país que termina por

mericana de la Salud, sobre la base de las estimaciones más recientes para cada país.

se observa en Nicaragua $(1,4 \%$ frente a $2,6 \%$, respectivamente, al umbral de pobreza relativa). Sin embargo, el incremento en la brecha de pobreza es casi 1,5 veces más alto en Paraguay. Esto indica que, en promedio, el GBS es más elevado en Paraguay que en Nicaragua para los grupos más empobrecidos de la población, que hay más pobres que gastan en salud de su propio bolsillo en Paraguay que en Nicaragua, o ambas cosas.

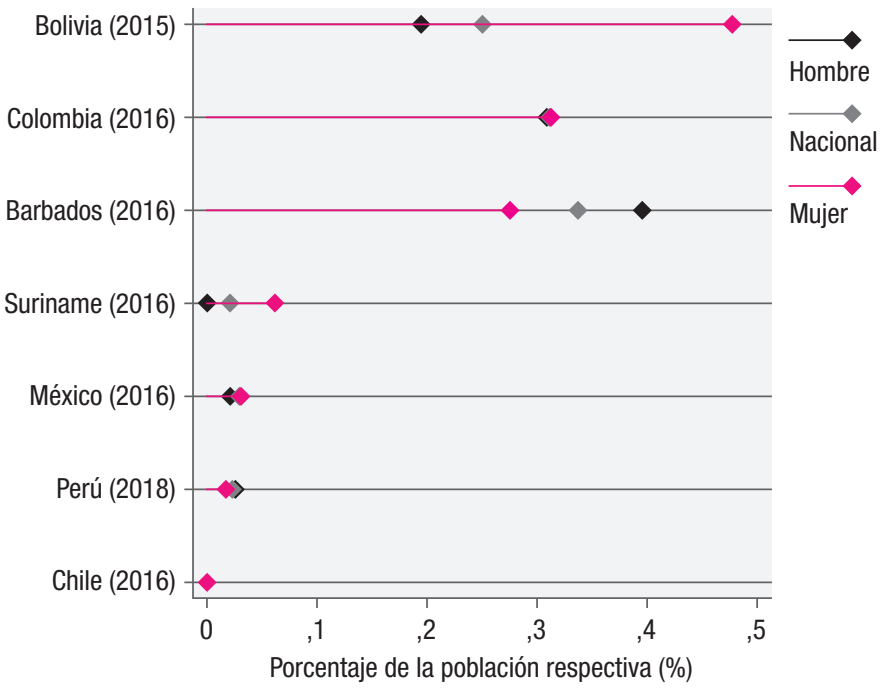

Nota: el umbral de 1,90 dólar estadounidense por día se mide en paridad de poder adquisitivo, el equivaente en moneda local se encuentra en la base de datos PovcalNet del banco mundial (actualización de marzo de 2019). La incidencia indica el porcentaje de la población en cada país que termina por debajo del umbral de pobreza absoluto extremo por causa del gasto directo en salud.

fuente: elaborada a partir de datos producidos por la Organización Panamericana de la Salud, sobre la base de las estimaciones más recientes para cada país.

\section{Protección financiera: desigualdades según el género y la medida bienestar de los hogares}

En la totalidad de los casos presentados en la figura 3a, con excepción de Barbados, el gasto catastrófico en salud afecta más a los hogares con mujeres como jefas de familia que a aquellos con hombres como jefes de hogar, cuando se considera un umbral de $10 \%$. Lo mismo ocurre con el gasto empobrecedor cuando el umbral es de 1,9 dólares, allí Bolivia se incorpora al grupo con mayor brecha de género (figura $3 b$ ). No obstante, cuando el umbral de gasto empobrecedor en salud es el de pobreza relativa (figura 3c), entonces predomina el efecto mayor sobre los hogares con hombres como jefes de hogares (con excepción de Barbados y Barbados).

Esta combinación de resultados podría explicarse por el nivel de pobreza absoluta mayor de los hogares con mujeres como jefas de hogar, dada la sensibilidad mostrada a los umbrales más bajos.

Por otro lado, por lo general, son las personas que viven en hogares pertenecientes a los primeros quintiles de consumo total, las más afectadas por el gasto empobrecedor en salud. Para que alguien del tercer o cuarto quintil sufra GBS empobrecedores, tiene que registrar un nivel de GBS muy alto.

Considerando el umbral de pobreza relativa, el gasto empobrecedor se encuentra en los tres primeros quintiles y predomina en el quintil 2 (figura 4a), con una tasa entre 4,3 y 5,0 veces más alta que la nacional, excepto en Barbados. La incidencia es notablemente alta en Ecuador y Nicaragua, donde entre $12 \%$ y $14 \%$ de la población del segundo quintil se empobrece por GBS.

Existe una coincidencia entre un mayor impacto promedio de los gastos empobrecedores en salud y la mayor variabilidad de 
FIGURA 4. Desigualdades en el empobrecimiento debido al gasto en salud en 10 países de América Latina y el Caribe, por quintil de consumo total del hogar. A: incidencia del gasto empobrecedor en salud por quintil. B: incremento en la profundidad de pobreza debido a gastos de bolsillo en salud por quintil.
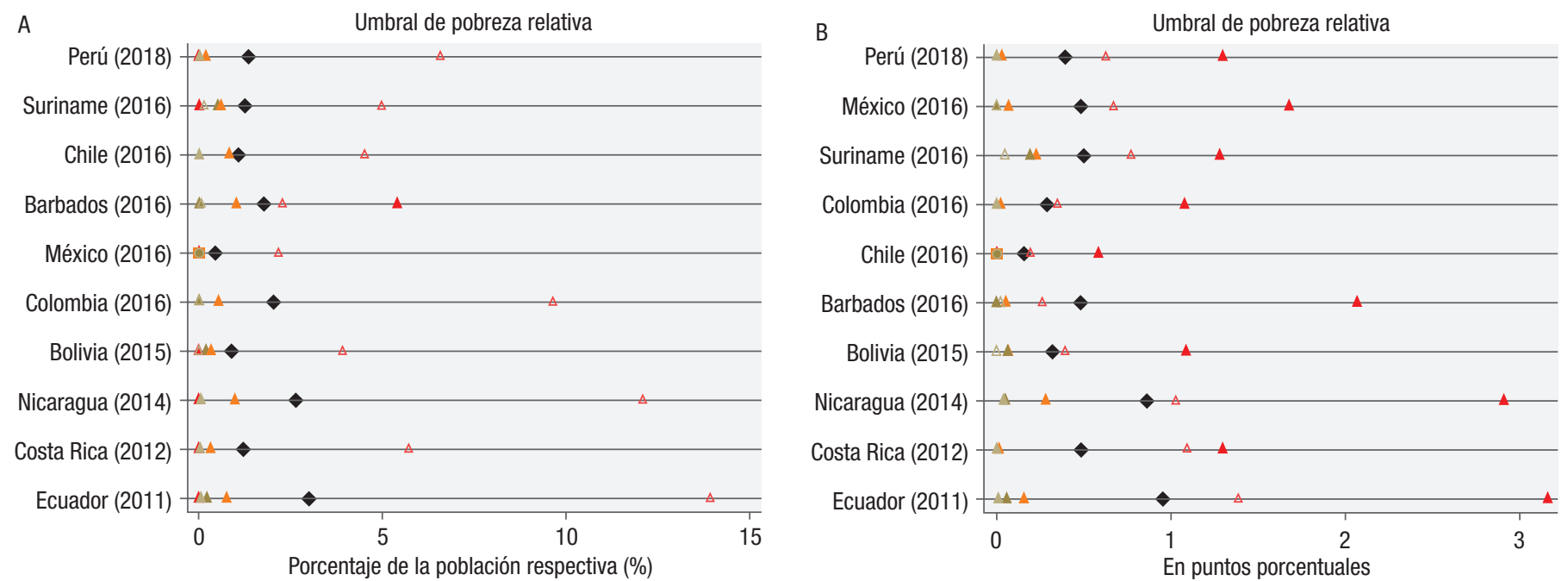

Notas: El umbral de pobreza relativa corresponde al $60 \%$ de la mediana del consumo total de los hogares por día en cada país. La incidencia indica el porcentaje de la población en cada país que termina por debajo del umbral de pobreza relativa por causa del gasto directo en salud.

Referencias: triángulo rojo: quintil 1; triángulo rojo vacío: quintil 2; cuadrado: quintil 3; círculo: quintil 4; círculo vacío: quintil 5; rombo negro: consumo nacional per cápita. Algunos hogares pueden parecer más ricos de lo que son porque han obtenido préstamos de dinero para financiar sus gastos directos en salud (u otros artículos) pero se puede suponer, con seguridad, que los hogares del quintil más bajo son realmente pobres. Fuente: elaborada a partir de datos producidos por la Organización Panamericana de la Salud, sobre la base de las estimaciones más recientes para cada país.

desigualdad entre los quintiles respecto del indicador. De este modo, Ecuador y Nicaragua (figura 4c) presentan los mayores impactos y las mayores desigualdades en los primeros quintiles, con diferencias de casi 10 puntos porcentuales respecto de las medias nacionales cuando se usa el umbral de pobreza relativa. México presenta los menores impactos y las distribuciones menos desiguales.

El impacto del GBS en los más pobres se obtiene usando el indicador de incremento en profundidad de pobreza. La figura $4 \mathrm{~b}$ muestra que, en todos los países, el incremento de la profundidad de pobreza debido al GBS es más alto en el primer quintil que en el segundo quintil. En el primer quintil varía entre 0,6 puntos porcentuales en México a alrededor de 3,0 puntos porcentuales en Nicaragua y Ecuador. En el segundo quintil, varía entre 0,2 puntos porcentuales en México y 1,4 en el caso de Ecuador. La desigualdad en el aumento de la profundidad de pobreza debido al GBS entre el primer quintil y el segundo quintil es más importante en Barbados, Chile, Ecuador y Nicaragua.

\section{Dinámica de la protección financiera y la cobertura de servicios de los sistemas de salud}

En la Región, el GBS es alto, con una deficiencia crónica de gasto público para financiar el sistema de salud, situación que tiene implicancias sobre la protección financiera $(21,22)$ (véanse las figuras b1 y b2 en el material suplementario). No obstante, existen países con similares niveles de GBS que presentan diferencias en los indicadores de protección financiera.

Al analizar la información de protección financiera y de cobertura de servicios, se reflejan posibles trayectorias del financiamiento en los países. Cuando se acepta este análisis bidimensional, la figura 5a muestra las posiciones de los países en el año más actual posible y las medianas regionales.
Se aprecian tres situaciones: los países en verde, que presentan mejores índices de cobertura de servicios y protección financiera (como El Salvador o México), la zona naranja con niveles intermedios ya sea equivalentes o con énfasis en una de las dos categorías (como Bolivia o Colombia); por último, están los casos en rojo que muestran una alta desprotección financiera y una baja cobertura de servicios (como Chile y Haití).

Incorporando varios años, al comparar los indicadores para Bolivia y Perú, se obtienen dos formas posibles de cambio en el tiempo (figura 5b). Bolivia disminuyó su indicador de gasto catastrófico de manera notable y en forma constante entre 2000 y 2012, logrando ubicarse por debajo de la mediana regional en 2008, con poco avance en cobertura de servicios. Luego avanza en cobertura de servicios en las siguientes mediciones (entre 2008 y 2015), y baja todavía un poco el gasto catastrófico. Sin embargo, ante un aumento importante de cobertura de servicios, se produce un aumento del gasto catastrófico en 2015.

En el caso de Perú, que inicialmente presentaba niveles de desprotección financiera similares a Bolivia, pero con mayores niveles de cobertura de servicios, la caída del gasto catastrófico es menos clara entre 2000 y 2013; pero después, entre 2013 y 2017 se produce una caída más categórica de gasto catastrófico, aunque mucho menor que en Bolivia, con aumento moderado también de la cobertura de servicios, y logra superar levemente la mediana regional en 2014.

En otros casos, sin la abundancia de puntos de medición de Bolivia y Perú, aun se pueden describir movimientos. Por ejemplo, Colombia disminuyó el gasto catastrófico en salud desde niveles muy altos, sin llegar a estar por debajo de la mediana regional y aumentó la cobertura de servicios levemente entre cuatro mediciones (2008-2016) sobrepasando la mediana regional. Chile retrocede de manera importante un gasto catastrófico que al inicio ya era bastante alto, con 
FIGURA 5. Gasto catastrófico en salud y cobertura de acceso a servicios de salud para el año más reciente disponible para 16 países y evolución en el tiempo para la Región y para cinco países en particular

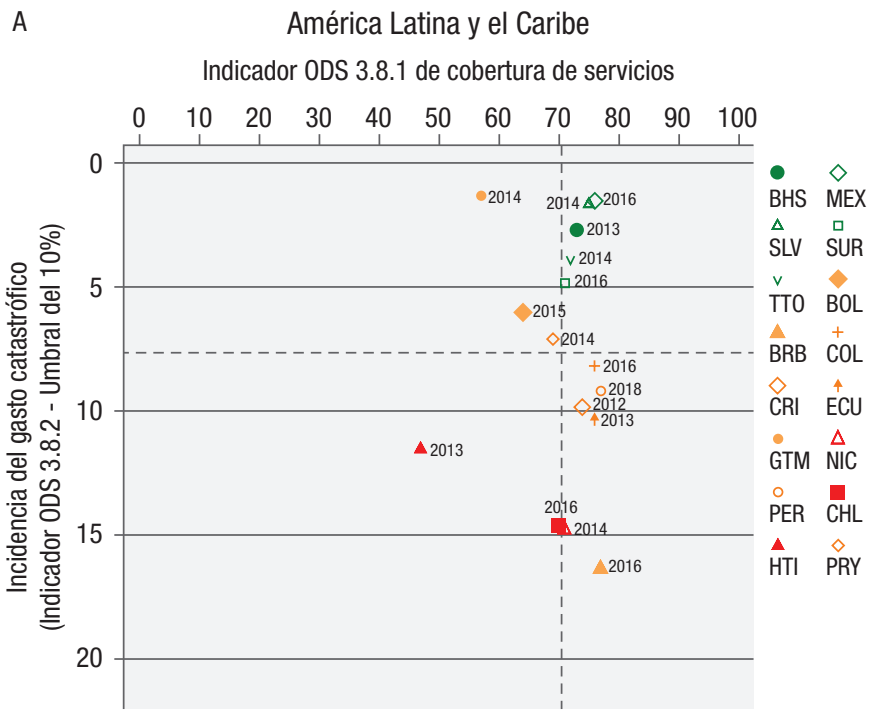

B

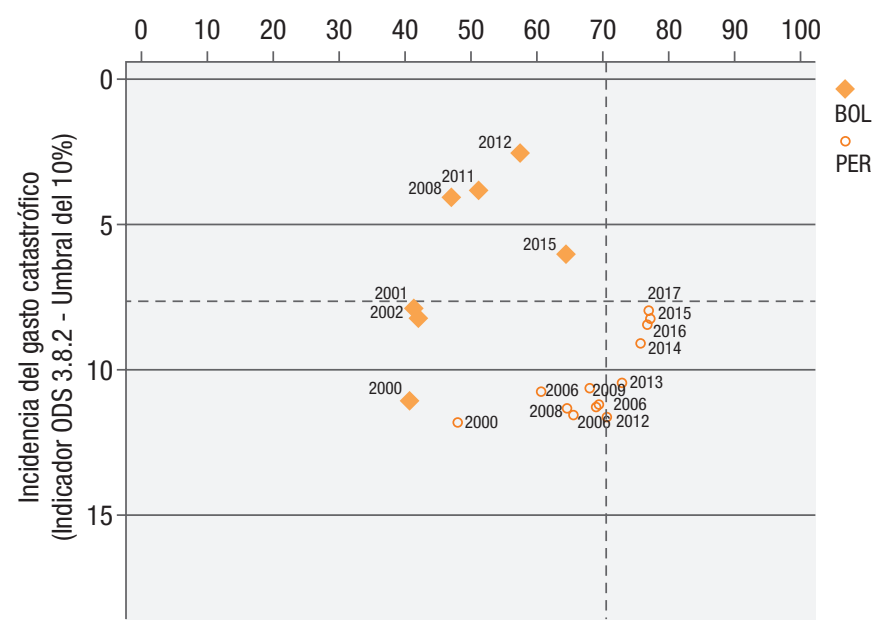

C

Chile, Colombia y México Indicador ODS 3.8.1 de cobertura de servicios

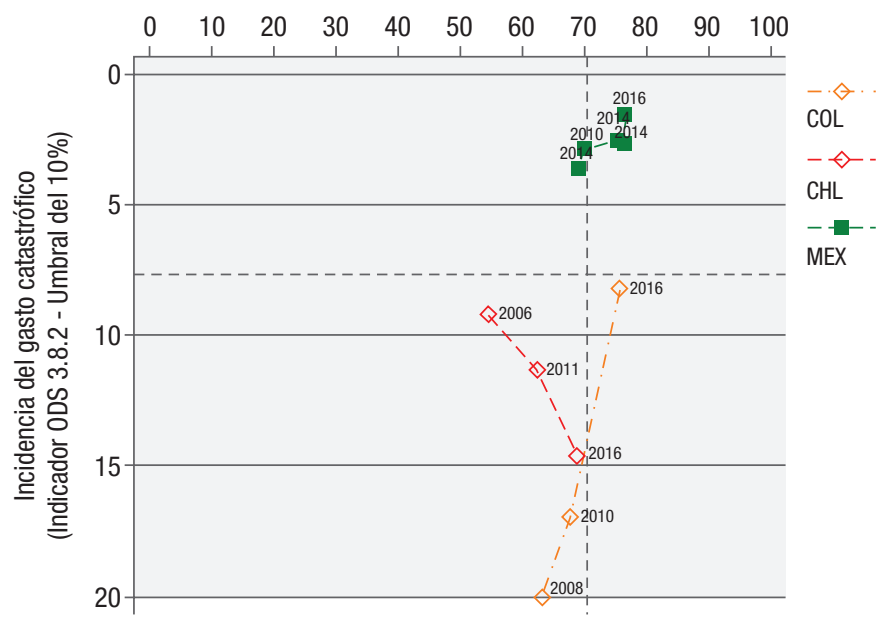

Nota: los cuadrantes se dividen por las medianas regionales no ponderados de los ODS 3.8.2 (umbral de 10\%) y 3.8.1 ODS. El año indicado corresponde a la estimación disponible más reciente para el ODS 3.8.2. Para el ODS 3.8.1, se usa la estimación más cercana a ese año.

bases de datos mundial de la Organización Mundial de la Salud (OMS) sobre la cobertura de servicios para el indicador ODS 3.8.1, y de la OMS y el Banco Mundial para el ODS 3.82 (actualizaciones del 2019).

ganancias moderadas de cobertura de servicios que no llegan a superar la mediana de ALC. México, por su parte, ya en 2008 se encontraba sobre las medianas regionales de cobertura de servicios y protección financiera, y se mueve con mejoramientos leves en ambas variables por un extenso período (figura 5c).

\section{DISCUSIÓN}

Las limitaciones de este trabajo se asocian con las diferencias entre las encuestas de los países, como el año de la misma y la técnica de consulta para obtener la información, de modo que las comparaciones pueden verse relativizadas. Aun así, se utilizan metodologías de estandarización que tratan de capturar algunas de las diferencias y que dan cuenta de las mejores prácticas disponibles a nivel internacional.

El GBS es alto y diferenciado en los países de la Región, y su impacto sobre el consumo total de los hogares es relevante. Existen países donde está presente en menos hogares, combinado con una participación alta en el gasto total en salud nacional, lo que implica que debiera estar concentrado en quienes acceden a servicios pagando montos relativamente elevados. En general, los países con GBS más extendido entre la población coinciden con un alto impacto en el consumo familiar y con indicadores de protección financiera también deficiente. El GBS ocurre, en su mayoría, en medicamentos, lo que tiende a generalizar hallazgos de otros trabajos (23). 
Dado el impacto del GBS en los hogares, la Región presenta importantes problemas de protección financiera, con efectos diferentes entre los países. Es posible identificar un grupo de mayores dificultades en cuanto al nivel y distribución de gasto catastrófico y empobrecedor (Barbados, Chile, Ecuador, Haití y Nicaragua), así como un grupo con ventajas relativas en protección financiera (Bahamas o El Salvador). También es posible identificar un grupo intermedio (Bolivia, Colombia y Perú). Algunos países presentan unos niveles positivos de protección financiera que resultan contraintuitivos por sus altos GBS en el financiamiento del sistema de salud (Guatemala y México).

Los gastos catastróficos y empobrecedores pueden afectar más a los hogares con mujeres como jefas de familia cuando se considera el umbral más exigente, pero este resultado no predomina cuando el umbral es el de pobreza relativa. Ello representa problemas de equidad y debe explicarse con el nivel de pobreza mayor de los hogares con mujeres como jefas de hogar.

La población de países más pobres no siempre se empobrece más por GBS en relación con otros países de menores niveles de pobreza; pero, en cualquier caso, el efecto que tiene el GBS en los hogares que ya son pobres es muy grave. Los hogares pertenecientes a los quintiles más bajos se ven más afectados por el gasto empobrecedor en salud y los países que se ven más afectados por este gasto, y también presentan mayor inequidad en la distribución del indicador entre los hogares. El incremento de la profundidad de pobreza debido al GBS es más alto en el primer quintil y existe una importante desigualdad en este aumento entre los primeros dos quintiles en países como Barbados, Chile, Ecuador y Nicaragua.

El financiamiento de los sistemas de salud se ha apoyado excesivamente en el GBS. Los tipos de reformas o estrategias de financiamiento propuestas por distintos países, junto con sus condiciones de base, forman parte de los niveles y diferencias entre los indicadores estudiados. De este modo, países que dependen de la misma manera del gasto en salud de los hogares pueden tener muy distintas incidencias de gasto catastrófico debido, en parte, al tipo y la manera en que esas políticas de acceso a los servicios de salud y su financiamiento son implementadas, en el contexto de unas estructuras determinadas del sistema de salud que, por lo general, están fragmentadas y segmentadas.

Al analizar la protección financiera cruzada con la cobertura de servicios, solo en algunos casos se pueden relacionar, de modo general y preliminar, los esfuerzos de fortalecimiento de los sistemas de salud en el tiempo, con series de datos que acompañan los períodos de implementación de políticas.

Por ejemplo, Bolivia, a partir de los 2000, introdujo programas de acceso a grupos poblacionales, como el Seguro Universal Materno Infantil, el Programa de Extensión de Cobertura a Áreas Rurales, el Modelo de Salud Familiar, Comunitaria e Intercultural, y el programa de subsidios monetarios Juana Azurduy dirigido a fortalecer la atención prenatal y del parto $(24,25)$. Además, hubo, por una parte un aumento importante del gasto público en salud, que pasó de $2,5 \%$ a $4,4 \%$ del producto interno bruto a partir del segundo decenio del siglo y, por otra parte, un planteamiento más reciente de conformación de un sistema único de salud (26).
Perú, por su parte, lanzó el seguro integral de salud (SIS) en 2002 con la integración de varios programas de cobertura. Impulsó el llamado "aseguramiento universal" desde 2009 con incrementos importantes en la adscripción al SIS, que adquirió un nuevo impulso a partir de la última reforma de 2013 (27), junto con un aumento del gasto público, pero en forma menos pronunciada que el caso anterior. En ambos casos, se lograron caídas importantes en los GBS nacional, que lo llevaron por debajo del promedio regional con una participación de $23,2 \%$ y 29,2\%, respectivamente, en 2018.

En México, resulta interesante apreciar cómo el GBS, que es elevado (50\% del gasto total en salud en el año relevante), produce efectos moderados en los niveles de desprotección financiera, con resultados más o menos constantes de cobertura de servicios que lo encuadran en una posición positiva. Podría tratarse de una combinación de gasto público bajo en salud y cobertura financiera segmentada con extensión relativamente concentrada del GBS a los contingentes sin cobertura de aseguramiento y del seguro popular, con énfasis en el gasto en medicamentos (28).

Para los demás países, se pueden describir algunas cuestiones básicas. Por ejemplo, en el caso de Chile, se aprecia un retroceso en protección financiera y poco avance en la cobertura de servicios. Se conoce la excesiva segmentación del sistema, así como el papel del sector privado, con dificultades en la protección financiera de medicamentos y en la atención ambulatoria $(29,30)$. Colombia, por su parte, parece un caso inverso al de México, ya que presenta gasto de bolsillo bajo como proporción del gasto nacional en salud, pero con indicadores de gasto catastrófico por sobre las medianas de la Región, aun cuando vienen disminuyendo en el tiempo.

Un análisis más profundo de las relaciones anteriores, que permita informar resultados ante procesos de implementación de reformas al financiamiento, permanece como desafío para futuras investigaciones.

Por último, una recomendación general que surge de este análisis es que el financiamiento de la salud debe apoyar el objetivo de brindar acceso equitativo a los servicios de salud necesarios y de calidad para la población. Para avanzar hacia la eliminación del GBS que impide esta meta y aumentar la protección financiera, se requiere de nuevos recursos públicos mancomunados de forma solidaria que lo reemplacen. El contexto actual de crisis y pandemia, aún en desarrollo, hace más patente la necesidad de un compromiso de los países con salud como prioridad fiscal, que permita un financiamiento público suficiente de sistemas de salud en transformación hacia la salud universal.

Contribución de los autores Todos los autores concibieron el estudio original. CCP y GFS recopilaron los datos y redactaron el manuscrito. Todos los autores analizaron los datos e interpretaron los resultados. Todos los autores revisaron el manuscrito y revisaron y aprobaron la versión final.

Conflicto de intereses Ninguno declarado por los autores.

Declaración Las opiniones expresadas en este manuscrito son únicamente responsabilidad de los autores y no reflejan necesariamente los de la Revista Panamericana de Salud Pública o la Organización Panamericana de la Salud. 


\section{REFERENCIAS}

1. Organización Mundial de la Salud, Banco Mundial. Global monitoring report on financial protection in health 2019. Ginebra: OMS, BID, BM; 2020. Disponible en: https://www.who.int/healthinfo/ universal_health_coverage/report/fp_gmr_2019.pdf?ua=1

2. Organizaicón Mundial de la Salud. Primary health care on the road to universal health coverage: 2019. Ginebra: OMS; 2019. Disponible en: https://www.who.int/healthinfo/universal_health_coverage/ report/uhc_report_2019.pdf

3. Organización Panamericana de la Salud. Resolución CD 53 R14. Estrategia para el acceso universal a la salud y la cobertura universal de salud. 54 Consejo Directivo, 66 sesión del comité regional de la OMS para las Américas, 29 de septiembre al 3 de octubre de 2014. Washington D.C.: OPS; 2014.

4. Naciones Unidas. Transformar nuestro mundo: la Agenda 2030 para el Desarrollo Sostenible Resolución aprobada por la Asamblea General el 25 de septiembre de 2015. Nueva York: ONU; 2015. Disponible en: https://unctad.org/system/files/official-document/ ares70d1_es.pdf

5. Organización Panamericana de la Salud.. Compact $30 \bullet 30 \bullet 30$ PHC for universal health. Washington, D.C.: OPS; 2019.

6. Organización Panamericana de la Salud. Salud Universal en el siglo XXI: 40 años de Alma-Ata". Informe de la Comisión de Alto Nivel. Edición revisada. Washington, D.C.: OPS; 2019.

7. Organización Panamericana de la Salud. Plan estratégico de la Organización Panamericana de la Salud 2020-2025: la equidad, el corazón de la salud, Washington D.C.: OPS; 2019. Disponible en: https://iris.paho.org/handle/10665.2/52717.

8. Organización Mundial de la Salud. Financiación de los sistemas de salud: el camino hacia la cobertura universal. Informe sobre la salud en el mundo 2010, Ginebra: OMS; 2010.

9. Tanahashi T. Health service coverage and its evaluation. Bulletin of the World Health Organization. 1978;56(2):295-303.

10. Báscolo E, Houghton N, Del Riego A. Leveraging household survey data to measure barriers to health services access in the Americas. Rev Panam Salud Publica. 2020;44:e100. https://doi.org/10.26633/ RPSP. 2020.100

11. Houghton N, Bascolo E, del Riego A. Monitoring access barriers to health services in the Americas: a mapping of household surveys. Rev Panam Salud Publica. 2020;44:e96. https://doi.org/10.26633/ RPSP.2020.96

12. Xu K, E. D. Household catastrophic health expenditure: a multicountry analysis. Lancet. 2003;362:111-7.

13. Organización Panamericana de la Salud. Protección financiera en salud en las Américas: mediciones de gastos catastrófico y gasto empobrecedor. Washington D.C.: OPS, en prensa; 2021.

14. Wagstaff A, Flores G, Smitz MF, Hsu J, Chepynoga K, Eozenou P. Progress on impoverishing health spending: results for 122 countries. A retrospective observational study. Lancet Global Health. 2018;6(2):e180-e192.

15. Foster JE, Greer J, Thorbecke E. A class of decomposable poverty measures. Econometrica, 1984;52(3):761-66.

16. Banco Mundial. Monitoring global poverty: report of the Commission on Global Poverty. Washington, D.C.: Banco Mundial; 2017.

17. Jolliffe D, Prydz EB. Estimating international poverty lines from comparable national thresholds. The Journal of Economic Inequality, 2016;14(2): 185-98.
18. Banco Mundial. Financial protection in health global monitoring data-2019. Universalhealth coveragedata. Washington,D.C.:Banco Mundial; 2019. Disponible en: https://datacatalog.worldbank. org/dataset/financial-protection-health-global-monitoringdata-2019

19. Organizaicón Mundial de la Salud. Financial protection. Universal health coverage data portal. Disponible en: https://www.who.int/ data/gho/data/themes/topics/financial-protection

20. Organización Mundial de la Salud.. Global Health Expenditure Database. Disponible en: https://apps.who.int/nha/database

21. Cid C, Pescetto C, Pagano J, Del Riego A, Fitzgerald J. Health financing and financial protection in the Americas. Working paper prepared by the WHO Regional Office for the Americas Universal Health Coverage Forum. Tokio: OMS; 2017.

22. Cid Pedraza C, Pagano JP, Pescetto C, Prieto L. Espacio fiscal para el financiamiento sostenible de los sistemas de salud y la salud universal. Rev Panam Salud Publica. 2018;42:e197. https://doi. org/10.26633/ RPSP.2018.197

23. Perticara M. Incidencia de los gastos de bolsillo en salud en siete países latinoamericanos. Santiago: CEPAL; 2008.

24. Ledo C, Soria R. Sistema de Salud de Bolivia. Salud Publica Mex. 2011;53:s109-s119.

25. Prieto L, Cid C. Análisis del sector salud de Bolivia. Notas técnicas 163. División de la Protección Social en Salud. Washington, D.C.: BID; 2010.

26. EstadoPlurinacional de Bolivia. Ley $N^{\circ} 1152$ :Hacia el sistema únicode salud, universaly gratuito,20defebrerode2019.Disponibleen:https:// www.lexivox.org/norms/BO-L-N1152.xhtml?dcmi_identifier= BO-L-N1152\&format=xhtml

27. Eibenschutz C, Saco Valdivia A, Tamez González S, Zafra Gatica X, Ramírez Villegas R. Reflexiones sobre el proceso de reforma sanitaria (1993-2013) y participación social en Perú. Saúde Debate. 2014;38(103):872-885.

28. Senado de la República de México. Iniciativa 2019 que reforma la Ley General de Salud, de la Ley de Coordinación Fiscal y de la Ley de los Institutos Nacionales de Salud en materia de acceso a los servicios de salud y medicamentos asociados para las personas que no poseen seguridad social. Gaceta de la Comisión Permanente. Ciudad de México: Senado de la República; 2019. Disponible en: http://infosen.senado.gob.mx/sgsp/gaceta/64/1/2019-07-03-1/ assets/documentos/Ini_Delgado.pdf

29. Cid C, Uthoff A. La reforma a la salud pendiente en Chile: reflexiones en torno a una propuesta de transformación del sistema. Rev Panam Salud Publica. 2017;41:e170. doi: 10.26633/RPSP.2017.170.

30. Frenz P, Siches I, Aguilera X, Arteaga O, Cid C, Estay R, et al. Propuesta para una reforma integral al financiamiento de la salud en Chile. Santiago de Chile: Escuela de Salud Pública Universidad de Chile y Colegio Médico de Chile; 2018.

Manuscrito recibido el 3 de febrero de 2021. Aceptado, tras revisión, para su publicación el 19 de mayo de 2021. 


\section{Sustainable Development Goals: impact of lack of financial protection in health in Latin American and Caribbean countries}

ABSTRACT Objective. To determine the impact of household out-of-pocket health spending, financial protection levels, and their inequality according to relevant variables in the countries of the Region; to investigate their evolution and relationship with health system services utilization.

Methods. Comparative descriptive information was compiled on out-of-pocket spending, its incidence in the population, and its weight and contribution to household consumption. Financial protection indicators for the national level and their distribution by quintiles of total household consumption and by gender are presented, and compared to an indicator of service coverage.

Results. Out-of-pocket spending and financial protection indicators are deficient but demonstrate differences among countries. The composition of health expenditure is identified for a subset of countries and significant gradients are seen when inequalities are studied. Changes over time and possible associations with service coverage levels are shown for several cases.

Discussion. Lack of financial protection affects a large part of the population. Additionally, there are groups of countries with greater difficulties than others, where a preponderance of spending is on medicines, and there is greater exposure of groups in conditions of vulnerability, such as the poorest and women, indicating great inequity. Policies of some countries that can be associated with improvement in financial protection are identified. To replace out-of-pocket spending, which is a barrier to access, countries need to increase public spending by financing health systems undergoing transformation toward universal health.

Palabras clave Cost control; financial risk protection; catastrophic health expenditure; impoverishing health expenditure; healthcare financing.

\section{Objetivos de Desenvolvimento Sustentável: impacto da falta de proteção financeira em saúde nos países da América Latina e do Caribe}

RESUMO

Palavras-chave
Objetivo. Determinar o impacto dos gastos diretos em saúde nas famílias, os níveis de proteção financeira e sua desigualdade, de acordo com variáveis relevantes nos países da Região; investigar sua evolução e sua relação com a utilização dos serviços do sistema de saúde.

Métodos. Foram coletadas informações descritivas comparativas sobre gastos diretos em saúde, sua incidência na população e seu peso e composição no consumo familiar. Os indicadores de proteção financeira são apresentados em nível nacional, estratificados por quintis de consumo total das famílias e por gênero, e são comparados com um indicador de cobertura de serviço.

Resultados. Os indicadores de gastos diretos e proteção financeira são limitados, mas demonstram diferenças entre os países. Foi possível identificar a composição dos gastos com saúde para um subgrupo deles, observando-se gradientes significativos quando as desigualdades são estudadas. Em alguns casos, observam-se também mudanças ao longo do tempo e possíveis associações com os níveis de cobertura de serviço.

Discussão. A falta de proteção financeira atinge grande parte da população. Foram observados grupos de países com mais dificuldades do que outros, com preponderância de gastos com medicamentos e maior exposição de grupos em situação de vulnerabilidade, como os mais pobres e as mulheres, o que denota alto nível de iniquidade. Em alguns países foram identificadas políticas que podem estar associadas à evolução da proteção financeira. Para substituir os gastos diretos, que constituem uma barreira ao acesso, os países precisam aumentar o gasto público, financiando os sistemas de saúde numa transformação rumo à saúde universal.

Controle de custos; proteção contra riscos financeiros; gasto castrófico em saúde; gasto em saúde empobrecedor; financiamento da assistência à saúde. 